Preprint

UCRL-JC-149066

\title{
Fabrication of Luminescent Nanostructures by Dip-Pen Nanolithography
}

\author{
A. Noy, A.E. Miller, J.E. Klare, B.L. Weeks, B.W. Woods, \\ J.J. DeYoreo
}

This article was submitted to The International Society for Optical Engineering (SPIE) Annual Meeting 2002, Seattle, Washington, July 7-11, 2002

Lawrence

Livermore

National

Laboratory

\section{June 25, 2002}

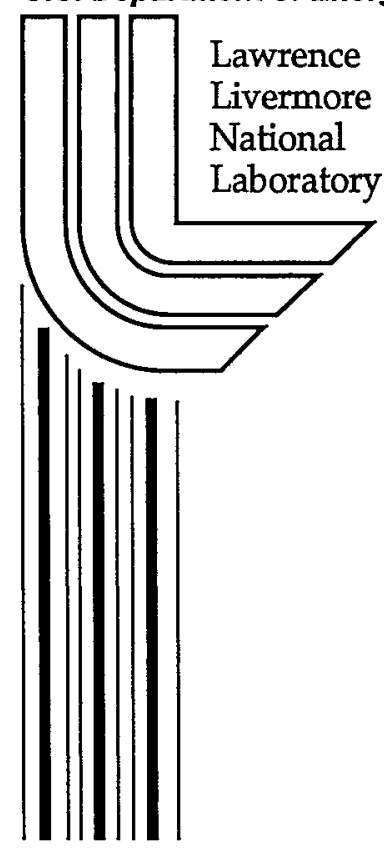




\section{DISCLAIMER}

This document was prepared as an account of work sponsored by an agency of the United States Government. Neither the United States Government nor the University of California nor any of their employees, makes any warranty, express or implied, or assumes any legal liability or responsibility for the accuracy, completeness, or usefulness of any information, apparatus, product, or process disclosed, or represents that its use would not infringe privately owned rights. Reference herein to any specific commercial product, process, or service by trade name, trademark, manufacturer, or otherwise, does not necessarily constitute or imply its endorsement, recommendation, or favoring by the United States Government or the University of California. The views and opinions of authors expressed herein do not necessarily state or reflect those of the United States Government or the University of California, and shall not be used for advertising or product endorsement purposes.

This is a preprint of a paper intended for publication in a journal or proceedings. Since changes may be made before publication, this preprint is made available with the understanding that it will not be cited or reproduced without the permission of the author.

This report has been reproduced directly from the best available copy.

Available electronically at http://www.doe.gov/bridge

Available for a processing fee to U.S. Department of Energy and its contractors in paper from

U.S. Department of Energy

Office of Scientific and Technical Information

$$
\text { P.O. Box } 62
$$

Oak Ridge, TN 37831-0062

Telephone: (865) 576-8401

Facsimile: (865) 576-5728

E-mail: reports@adonis.osti.gov

Available for the sale to the public from

U.S. Department of Commerce

National Technical Information Service

5285 Port Royal Road

Springfield, VA 22161

Telephone: (800) 553-6847

Facsimile: (703) 605-6900

E-mail: orders@ntis.fedworld.gov

Online ordering: http://www.ntis.gov/ordering.htm

OR

Lawrence Livermore National Laboratory

Technical Information Department's Digital Library

http://www.llnl.gov/tid/Library.html 




\title{
Fabrication of luminescent nanostructures by dip-pen nanolithography
}

\author{
Aleksandr Noy", Abigail E. Miller, Jennifer E. Klare, Brandon L. Weeks, \\ Bruce W. Woods, James J. DeYoreo \\ Material Science and Technology Division and BioSecurity Support Laboratory \\ Lawrence Livermore National Laboratory
}

\begin{abstract}
We used a combination of dip-pen nanolithography and scanning optical confocal microscopy to fabricate and visualize luminescent nanoscale patterns of various materials on glass substrates. We show that this method can be used successfully to push the limits of dip-pen nanolithography down to controlled deposition of single molecules. We also demonstrate that this method is able to create and visualize protein patterns on surfaces. Finally, we show that our method can be used to fabricate polymer nanowires of controlled size using conductive polymers. We also present a kinetic model that accurately describes the deposition process.
\end{abstract}

Keywords: dip-pen nanolithography, single molecules, polymer nanowires, deposition kinetics

\section{INTRODUCTION.}

Nanoscale optoelectronic devices are one of the most promising applications of nanotechnology. The task of creating such devices requires robust methods for controlled deposition of optically functional materials on surfaces. Popular deposition strategies include conventional lithography, soft lithography ${ }^{1}$ and molecular self-assembly. ${ }^{2}$ However, at the nanometer scale most of these methods either break down or are poorly controlled. In contrast, one method that is specifically designed to fabricate nanometer scale patters is dippen nanolithography (DPN) ${ }^{3,4}$ DPN uses an "inked" atomic force microscope (AFM) tip to deposit the ink material directly onto the surface in an arbitrary configuration, which is ideally suited for fabricating surface patterns in the laboratory setting. Recently, researchers used dip-pen nanolithography to fabricate patterns of chemical functionality, ${ }^{5}$ dendrimers, ${ }^{6}$ and raised gold wires. ${ }^{7}$

Ultimately, the limit to the feature size in DPN is determined by the probe geometry, water meniscus properties and the chemical nature of the ink. ${ }^{3,8}$ Currently, dip-pen nanolithography is restricted to creating patterns that AFM can visualize either by topographic imaging or by lateral force imaging. Therefore, to reveal the pattern we need to deposit material on the surface in the amount that is sufficient to produce the contrast in the AFM image. Thus, even if dip-pen nanolithography is capable of scaling down the amount and the surface concentration of the deposited material, the resulting patterns cannot be visualized by traditional AFM imaging.

We show that we can combine dip-pen nanolithography with single molecule optical detection to create and visualize nanoscale patterns of luminescent materials down to single-molecule deposition levels. We then demonstrate that the technique can be used to create protein patterns. Finally, as a first step towards creating optoelectronic devices we use dip-pen nanolithography to fabricate light-emitting polymer nanowires of predetermined size.

\section{EXPERIMENTAL.}

2.1 Substrate preparation and chemicals. Round cover glasses (Fisher Scientific) were cleaned by sonicating for $20 \mathrm{~min}$ in $2 \%$ solution of Helmanex cleaner (Hellma, Germany) and then rinsed with copious amounts of de-ionized water (Millipore). They were then sonicated for 10-20 min in de-ionized water, rinsed and stored under water until use. Gold films on mica substrates were purchased from Molecular Imaging and used as received. All chemicals were used as received without any further purification. 
Mercaptohexadecanoic acid (Aldrich) was dissolved in ethanol to $10^{-3} \mathrm{M}$ concentration. Rhodamnime 6G (Molecular Probes, Eugene, OR) was dissolved in methanol to $10^{-7} \mathrm{M}$ concentration. C. Hollars (LLNL) generously donated HCG antibody tagged with tetramethylrhodamine (TMR) fluorescent dye. $1 \mathrm{mg} / \mathrm{mL}$ solution in PBS buffer of TMRlabeled HCG antibody was used for inking. Poly[2-methoxy5-2'-ethylhexyl)oxy-1,4-phenylenevinylene] (MEH-PPV) polymer was available from previous studies. ${ }^{9}$ Prior to inking the polymer was dissolved in chloroform to $10^{-7} \mathrm{M}$ concentration.

2.2 Instrumentation. Our instrument combines atomic force microscopy and scanning confocal microscopy functionalities. A Bioscope AFM head (Digital Instruments, Santa Barbara, CA) is mounted on a Nikon Eclipse 300 inverted microscope equipped with a custom-built stage that incorporates a closedloop piezo scanner (PolytecPI,Germany). The scanning stage is used in both the lithography procedure and confocal imaging. The fluorescence excited by a 514 line of Ar-Ion laser (Coherent Innova-70) is collected with $100 \times 1.4 \mathrm{NA}$ lens, passed through a $50 \mathrm{~mm}$ pinhole to reject stray light and then detected by an avalanche photodiode (Perkin Elmer Optoelectronics, Santa Clara, CA). Excitation light was rejected by a long-pass filter (Omega Optical, VT).

\section{RESULTS AND DISCUSSION}

3.1 Controlled deposition of single molecules. Conventional dip-pen nanolithography with alkanethiol inks relies on the formation of a chemical bond between the ink and the substrate to facilitate the ink transfer from the tip to the sample surface. However, recent works demonstrated that even weaker interactions such as van-der-Waals interactions are often sufficient to facilitate the transfer of matter from the tip to the sample. 6.7 We have used dilute solutions of Rhodamine 6G (R6G) dye as the writing "ink" to create luminescent nanoscale patterns. A drop of the $10^{-7} \mathrm{M}$ R6G solution in methanol was absorbed onto a thin paper membrane. We then gently lowered the AFM tip into contact with the paper for 30-60 seconds using a custom-built translation stage under the optical microscope. Lithography was performed immediately after the "inking". R6G has strong affinity for negatively charged glass surfaces; therefore we did not use any surface treatments to promote the transfer of the "ink". All patterns were fabricated at ambient humidity. Both fabrication and subsequent imaging of the patterns was carried out using a custombuilt instrument (Figure 1) that combines atomic force microscopy and scanning confocal microscopy functionalities. ${ }^{10}$

Figure 2A shows a fluorescent confocal microscopy image of a pattem of R6G deposited on glass. Several features of this image are noteworthy. First, the apparent width of the lines in the image does not reflect the actual line width of the deposited dye. Rather, image linewidth is determined by the size of the confocal microscope probing beam (in our case $-600 \mathrm{~nm}$ ). Second, several features in the optical image do not show a continuous line, but rather are composed of a number of spots. Moreover, these spots showed features that are characteristic of a single molecule emission pattern, such as a striped pattern and abrupt emission cessation that is indicative of a dye molecule going in and out of dark states and finally photobleaching. ${ }^{11}$ Likewise, as we continued to image our pattern, it rapidly degraded from photobleaching. From these observations we concluded that our pattern was composed of a number of isolated single molecules deposited in a straight line by the AFM tip. Notably, we did not observe any evidence of the pattern in normal or lateral force AFM images. Therefore, the amount of the material deposited on the surface was not sufficient to cause a height change or an appreciable surface free energy change. This observation is also consistent with a pattern that is composed of only a number of single molecules. It is impossible to say how well the single molecules are aligned within the pattern line since the optical image does not provide nearly enough resolution to answer this question. However, we can speculate that the dye molecules are confined laterally at least within the typical line width attainable with alkanethiol dip-pen nanolithography. 
Such width is $\sim 30 \mathrm{~nm}$ in our case, but other groups have reported the patterns with line widths as small as $15 \mathrm{~nm}^{3}$ However, this limit may simply reflect the fact that lateral force microscopy cannot visualize thiol lines smaller than $15 \mathrm{~nm}$, even if they can be fabricated by dip-pen nanolithography. Changing the concentration of the dye solution used for inking along with careful adjustment of ambient humidity can control the average spacing between the molecules along the written line. For our sample we estimated this spacing to be $566 \pm 210 \mathrm{~nm}$. We expect that further improvements in the deposition process such as smaller probes, rigorous humidity control and careful choice of the writing ink will result in smaller line widths and more regular deposition patterns. These improvements will permit better alignment of single molecules on the sample for studies of the proximity effects in single molecule interactions.

3.2 Protein patterns. One of the promising potential applications of nanolithography is fabrication of ultra-high density protein patterns for use in "biochips". ${ }^{12}$ Some of the current approaches use soft lithography ${ }^{12}$ or "nanografting"13 to create reactive sites in a self-assembled monolayer. Proteins then attach to these reactive sites via a chemical reaction. Both of these methods are complex multi-stage processes and use chemical attachment, which is always complicated by the problem of non-specific adsorption. Dip-pen nanolithography provides a simple and straightforward way for creating protein patterns since features can be directly written onto the surface. Significantly, this method avoids the complications associated with non-specific binding. If the protein is tagged by a specific fluorophore, then the pattern can be easily identified and verified using optical imaging. To demonstrate the feasibility of this approach we fabricated patterns of human chorionic gonadotropin (HCG) antibody tagged with tetramethylrhodamine (TMR) dye on the glass surface (Figure 2B). Glass surface was pre-treated with 3-glycidoxypropyltrimethoxysilane to introduce the epoxy groups that facilitate protein adhesion to the surface. Reliable fabrication of protein chips will require the ability to deposit proteins at high surface concentration. To demonstrate this capability, we used a high concentration of the protein "ink" $\left(\sim 2 \cdot 10^{-5} \mathrm{M}(1 \mathrm{mg} / \mathrm{ml})\right.$ solution in PBS buffer) for fabrication of the pattern in Figure 2B. In addition each line in the pattern was overwritten 10 times to enhance the surface concentration of the protein. The lines in the optical image on the Figure 2B appear continuous and no sufficient photobleaching was observed during scanning. The absence of single molecule behavior suggests that we have achieved large protein density in our lines.

In addition, optical detection of a written pattern provides a straightforward way to generalize the process for creating patterns that incorporate many different proteins. Such patterns can be successfully fabricated and imaged by using different protein inks tagged with different fluorophores in a manner analogous to dippen nanolithography with multiple inks. ${ }^{5}$

3.3 Fabrication of polymer nanowires. We have used our apparatus to fabricate and image nanowires of luminescent conductive polymers. These materials have long been under investigation as potential components of polymer LEDs ${ }^{14}$ and fabrication of nanometer scale polymer structures is a potentially significant step for creating nano-LED device prototypes. In addition, nanowires of these polymers present an ideal system for studying photoluminescence and electron transport in organic one-dimensional materials. Polymer nanowires have previously been fabricated using template-directed synthesis ${ }^{15}$ and stretching of polymer bridges. ${ }^{16}$ However, these methods do not allow good control over the size and position of a nanowire, or any way to assemble these nanowires in a predetermined configuration. Dip-pen nanolithography with its ability to create surface patterns is ideally suited for solving these problems. We 
demonstrated controlled deposition procedure by writing a series of polymer lines on a glass surface (Figure 3A) using a $10^{-7} \mathrm{M}$ solution of poly[2methoxy-5-2'-ethylhexyl)oxy-1,4-

phenylenevinylene] (MEH-PPV) in chloroform as ink. Only the following three factors limit the length of these nanowires: (1) the available range of the force microscope scanner, (2) the amount of material on the probe tip and (3) the ability of the tip to deliver continuous ink flow. As in the two experiments that we discussed earlier, the apparent width of the structure in the image does not correspond to the real width of the nanowire; instead it is determined by the size of the confocal microscope excitation beam. Besides the tip size, the major variable in controlling the nanowire thickness is the tip translation speed. To verify this we created a series a series of the polymer lines (Figure $3 \mathrm{~A}$ ) using different tip speed to fabricate each line. We used the fluorescence intensity as a measure of the amount of material deposited in each line, since it is impossible to infer the line width from the optical image due to probing beam size limitation. We found that the amount of material deposited in the line during nanolithography decreases with the increase in tip translation speed (Figure 3B). To compare this system to a well-characterized system, we performed a similar measurement for dip-pen nanolithography using alkanethiol ink. Figure $3 \mathrm{C}$ shows a series of lines written on gold substrates using $10^{-3} \mathrm{M}$ mercaptohexadecanoic acid solution in ethanol as ink. We found that in this system the line thickness also increases as the probe translation speed decreases (Figure 3D). The observed trends confirm that at constant humidity the amount of material deposited on the surface is effectively controlled by the probe translation speed.

3.4 Ink transport kinetics. Ultimately, the amount of the material deposited on the sample is determined by the kinetics of the material transfer from the tip to the sample. We can describe it by a simple kinetic model. ${ }^{8}$ Qualitatively, the deposition involves several processes. When the "inked" AFM tip contacts the surface, ink molecules dissolve from the tip into the capillary meniscus, diffuse through the meniscus and then attach to the surface. After a time, $\tau$, the tip is removed from the surface. At this time, ink molecules are both attached to the surface and dissolved in the meniscus. Through diffusion, the dissolved thiols will eventually attach to the substrate, contributing to the final feature size. Regardless of the tip-meniscus geometry, the magnitude of the diffusion constant, or the dissolution rate, the total number of molecules, $\mathrm{N}$, transferred to the surface will be given by the integral of the transfer rate $\mathrm{dN} / \mathrm{dt}$ over the total tip-surface contact time $\tau$. Two terms contribute to $\mathrm{dN} / \mathrm{dt}$ : First, molecules dissolve from the tip through thermal activated detachment at a rate:

$$
\left(\frac{d N}{d t}\right)_{+}=\frac{A}{\pi a^{2}} \beta_{+}=\frac{A}{\pi a^{2}} v e^{-E_{D} / k T}
$$

where $A$ is the contact area between the tip and the meniscus, $\pi a^{2}$ is the average area per ink molecule on the tip, $\mathrm{E}_{\mathrm{D}}$ is the activation energy for ink detachment, $k$ is Boltzmann's constant, $T$ is the temperature, and $v$ is an attempt frequency. (Note that typically, the ink coverage on the tip is very high, therefore ink molecules are not detaching from the surface of a silicon nitride tip, rather they are detaching from the surface of a bulk ink solid that has precipitated onto the tip during the inking process.) Second, molecules return to the tip simply due to impingement and attachment at a rate given by: 


$$
\left(\frac{d N}{d t}\right)_{-}=A \beta C_{0} \approx A\left(\frac{k T}{2 \pi m}\right)^{1 / 2} e^{-E_{\Lambda} / k T} C_{0}
$$

where $m$ is the mass of an ink molecule, $E_{A}$ is the activation energy for attachment, and $C_{0}$ is the concentration of ink in solution adjacent to the tip. The far right hand side of Eqn. 2 assumes a gas kinetic expression for the impinging flux. A more complicated description would modify the factors in $\bar{\beta}^{-}$, but will not change the dependence on $\mathrm{C}_{0}$. (Note that in the absence of diffusion, $\mathrm{C}_{0}$ will simply increase until it reaches the equilibrium solubility, $\mathrm{C}_{e}$, at which time, $\left.(\mathrm{dN} / \mathrm{dt})_{+}=(\mathrm{dN} / \mathrm{dt})_{\text {. }^{-}}\right)$

Assuming the tip moves over a distance 1 at a speed $v$, we can use Equations 1 and 2 and integrate over the contact time to estimate the total number of molecules transferred to the sample:

$$
N=\int_{0}^{l}\left(\frac{d N}{d t}\right)\left(\frac{d t}{d x}\right) d x=\frac{1}{\mathrm{v}} \int_{0}^{l}\left(\frac{d N}{d t}\right) d x
$$

We also need to recognize that $\mathrm{C}_{0}$ is a function of $\mathrm{v}$, since the rate at which the tip moves over the surface controls the buildup of the ink molecules in the meniscus. Recognizing that $C_{0}(v)$ and $\beta$. are both independent of $x$ and that the line width $W=N \pi a^{2} / 1$, Equation 4 becomes:

$$
W=\left(\frac{A}{\mathrm{v}}\right)\left(\beta_{+}-\pi a^{2} \beta_{-} C_{0}(\mathrm{v})\right)
$$

The exact form of $\mathrm{C}_{0}$ depends on a number of factors including the tip-meniscus geometry, surface attachment probability and the diffusivity. However, if we consider that $\mathrm{C}_{0}$ must be small at high tip speeds, where the mass transfer from the tip to the surface is unimpeded by diffusion, two regimes emerge. For high $v, C_{0} \approx 0$ and the line width is given by the first term in Equation 4 , that is $W \sim A / v$. This is the regime in which surface detachment kinetics dominates the transfer process. For slow tip speeds, $\mathrm{CO}$ reaches a limiting value $C_{0}<C_{e}$. Once again, $W \sim A / v$, but the slopes of the linear relationship is reduced. In this regime the transfer process is controlled by diffusion. Therefore, as we increase the contact time of the tip with a particular surface region $(t \sim 1 / v)$, the line width initially increases linearly, but then slows down and transitions to a new, also linear, regime. Experimental dependencies of line width on $1 / \mathrm{v}$ that we obtained for both polymer and alkanethiol deposition (Figure 3B,D) clearly show the evidence for such slow-down. Therefore despite its simplicity, our model captures the dominant features of the DPN deposition kinetics in these systems.

Lastly, as evident from the image in the Figure 3A, sometimes during the polymer deposition the tip can "skip" and leave "blotches" of material on the sample. Alkanethiol ink typically does not exhibit such behavior mainly due to the smaller molecule size. Improved control over the tip chemical functionality and the inking process should give finer control over the ink detachment rate and minimize such pattern irregularities. Currently we are unable to verify the continuity of the nanowires or determine the alignment of the polymer chains on the wire. Further investigations of the electron transport properties and photoluminescent properties of these wires will provide answers to these questions.

\section{CONCLUSIONS.}

In summary, we have used dip-pen nanolithography combined with scanning confocal optical microscopy to create and image luminescent surface patterns of arbitrary shape. We demonstrated that this method affords a robust and versatile way to pattern a variety of photoluminescent materials on the surface. Significantly, we showed that dip-pen nanolithography could be used to deposit increasingly small amounts of material down to single isolated molecules. We also showed that a combination of dip-pen nanolithography and optical detection could be used to create and identify protein patterns on the surface, while avoiding the problem of non-specific adsorption. We demonstrated that dip-pen nanolithography affords a direct and flexible route to creating polymer nanowires of a predetermined size and shape on surfaces. We also presented a simple kinetic model that captures main physics of the deposition process. These results open up several possibilities for fundamental and applied research. Further refinements in the control of the spacing between isolated single molecules will present an ideal model system for studying proximity effects in the interactions between isolated fluorophores. The ability to fabricate arrays of single 
molecules with controlled spacing will allow studies of collective behavior in such systems. Patterning optically tagged proteins could provide a direct route to fabrication of prototype "biochips" once the protein surface attachment chemistry is refined. In addition to significant reduction in the feature size, dip-pen nanolithography simplifies fabrication process since it eliminates multiple protection/deprotection steps necessary for conventional lithographic manufacturing of such chips. Finally, fabrication of conductive polymer nanowires could open up numerous possibilities for fundamental studies of electron transfer and photoconductivity in such structures. In addition, fine control over the size and shape and position of these wires will greatly facilitate their incorporation into prototype optoelectronic devices.

\section{ACKNOWLEDGEMENTS.}

AN acknowledges LLNL Postdoctoral Fellowship. JEK acknowledges support from AWU Fellowship. We thank C. Hollars and T. Huser for generous donations of labeled HCG antibody and MEH-PPV polymer and for helpful discussions. This work was performed under the auspices of U.S. Department of Energy by the University of California, Lawrence Livermore National Laboratory under contract \#W-7405-Eng-48.

\section{REFERENCES}

Y. N. Xia, J. A. Rogers, K. E. Paul et al., Chemical Reviews 99 (7), 1823 (1999). D Philp and J F Stoddart, Angew. Chem. Int. Ed. Engl. 35, 1154 (1996).

R. D. Piner, Zhu Jin, Xu Feng et al., Science 283 (5402), 661 (1999).

C. A. Mirkin, Inorganic Chemistry 39 (11), 2258 (2000).

S. H. Hong, J. Zhu, and C. A. Mirkin, Science 286 (5439), 523 (1999).

J. Li, D. J. Qin, J. R. Baker et al., Macromolecular Symposia 167, 257 (2001).

B. W. Maynor, Y. Li, and J. Liu, Langmuir 17 (9), 2575 (2001).

BL. Weeks, A Noy, AE Miller et al., Phys. Rev. Letters, accepted.

T. Huser, M. Yan, and L. J. Rothberg, Proceedings of the National Academy of Sciences of the United States of America 97 (21), 11187 (2000).

A. Noy and T. R. Huser, paper in preparation.

S. Weiss, Science 283 (5408), 1676 (1999).

M. Mrksich and G. M. Whitesides, Trends in Biotechnology 13 (6), 228 (1995).

K. Wadu-Mesthrige, N. A. Amro, J. C. Garno et al., Biophysical Journal 80 (4), 1891 (2001).

R. H. Friend, R. W. Gymer, A. B. Holmes et al., Nature 397 (6715), 121 (1999);

J. H. Burroughes, D. D. C. Bradley, A. R. Brown et al., Nature 347 (6293), 539 (1990).

C. G. Wu and T. Bein, Science 264 (5166), 1757 (1994).

H. X. He, C. Z. Li, and N. J. Tao, Applied Physics Letters 78 (6), 811 (2001).

* Correspondence should be addressed to AN (noyl@llnl.gov), L-234, Lawrence Livermore National Laboratory, Livermore, CA 94538 